\title{
Spin-polarized ballistic conduction through correlated Au-NiMnSb-Au heterostructures
}

\author{
C. Morari, ${ }^{1}$ W. H. Appelt, ${ }^{2,3}$ A. Östlin, ${ }^{4}$ A. Prinz-Zwick, ${ }^{2}$ U. Schwingenschlögl, ${ }^{5}$ U. Eckern, ${ }^{2}$ and L. Chioncel ${ }^{3,4}$ \\ ${ }^{1}$ National Institute for Research and Development of Isotopic and Molecular Technologies, 67-103 Donat, 400293 Cluj Napoca, Romania \\ ${ }^{2}$ Theoretical Physics II, Institute of Physics, University of Augsburg, 86135 Augsburg, Germany \\ ${ }^{3}$ Augsburg Center for Innovative Technologies, University of Augsburg, 86135 Augsburg, Germany \\ ${ }^{4}$ Theoretical Physics III, Center for Electronic Correlations and Magnetism, Institute of Physics, \\ University of Augsburg, 86135 Augsburg, Germany \\ ${ }^{5}$ Physical Science and Engineering Division (PSE), King Abdullah University of Science and Technology (KAUST), \\ Thuwal 23955-6900, Saudi Arabia
}

(Received 26 September 2017; published 20 November 2017)

\begin{abstract}
We examine the ballistic conduction through $\mathrm{Au}-\mathrm{NiMnSb}-\mathrm{Au}$ heterostructures consisting of up to four units of the half-metallic NiMnSb in the scattering region, using density functional theory (DFT) methods. For a single NiMnSb unit the transmission function displays a spin polarization of around 50\% in a window of $1 \mathrm{eV}$ centered around the Fermi level. By increasing the number of layers, an almost complete spin polarization of the transmission is obtained in this energy range. Supplementing the DFT calculations with local electronic interactions, of Hubbard-type on the Mn sites, leads to a hybridization between the interface and many-body states. The significant reduction of the spin polarization seen in the density of states is not apparent in the spin polarization of the conduction electron transmission, which suggests that the hybridized interface and many-body induced states are localized.
\end{abstract}

DOI: 10.1103/PhysRevB.96.205137

\section{INTRODUCTION}

Multilayer heterostructures composed of alternating magnetic and nonmagnetic metals offer a large flexibility for tailoring spin-sensitive or spin-contrasted electron transport properties of spintronic devices. Highly spin-polarized materials such as half-metallic ferromagnets (HMF) are expected to play a crucial role [1,2]. The extreme spin polarization of half metals (i.e., 100\%) is a consequence of their band structure: These materials are metallic for one spin channel and insulating or semiconducting for the other. The prototype half-metallic material is the semi-Heusler compound $\mathrm{NiMnSb}$ [1].

High quality films of NiMnSb alloys were grown by molecular epitaxy [3], or magnetron sputtering [4], with the measured conduction electron spin polarization smaller than about 58\% [5]. This polarization value is consistent with measurements using electron tunneling techniques [6]. It was shown that during the growth of the NiMnSb thin films, first $\mathrm{Sb}$ and then $\mathrm{Mn}$ atoms segregate to the surface, decreasing the spin polarization [7]. By removing the excess $\mathrm{Sb}$ a nearly stoichiometric ordered alloy surface terminated by a MnSb layer is obtained, with a spin polarization of about $67 \pm 9 \%$ at room temperature [7-9]. Magnetic circular dichroism measurements showed a reduction of both the manganese and nickel moments around $80 \mathrm{~K}$. Borca et al. [9] concluded that at this specific temperature, a transition from a half-metallic to a normal ferromagnetic state takes place, and the magnetic coupling of the manganese and nickel moments is lost. The computational study of Ležaić et al. [10] suggests a vanishing of the $\mathrm{Ni}$ moment at around $80 \mathrm{~K}$ with a simultaneous loss of polarization. Studies on magnetic properties of ferromagnetic NiMnSb(100) [9] or NiMnSb(001) [11] interfaces are also available. The $80 \mathrm{~K}$ anomaly, however, is not reflected in the spontaneous magnetization of bulk $\mathrm{NiMnSb}$ [12]. An alternative scenario which addresses the contradiction between the theoretical predictions and the experimental results concerning the spin polarization in half metals is provided by finite-temperature effects [13] and nonquasiparticle (NQP) states [2,14-18].

Advanced materials with high-performance halfmetallicity are required for further improvements of currentperpendicular-to-plane giant magnetoresistance (CPP-GMR) devices. It has been shown that the CPP-GMR ratio is considerably reduced due to sample defects [19]. This indicates that a precise control over the sample purity and structure is highly desirable. Recent experiments [20] have been conducted on (001)-oriented fully epitaxial NiMnSb heterostructures with Ag spacer layers. A negative anisotropic magnetoresistance (AMR) ratio and small discrepancies of the AMR amplitudes between room temperature and $10 \mathrm{~K}$ were observed in a single epitaxial NiMnSb film, indicating robust bulk half-metallicity against thermal fluctuations. The modest CPP-GMR ratios were attributed to interface effects between $\mathrm{NiMnSb}$ and $\mathrm{Ag}$.

In this paper we address the question of ballistic conduction by examining the density of states (DOS) and the transmission in the vicinity of the Fermi energy $\left(E_{F}\right)$. The computational setup consists of $\mathrm{NiMnSb}$ units situated between $\mathrm{Au}$ leads, along the (001) direction. Au is a frequently used material for the leads and it has a lattice constant $(\approx 4.08 \AA)$ similar to $\mathrm{Ag}$ $(\approx 4.09 \AA)$. Therefore the lattice mismatch is only $\approx 2 \%$ so that fully epitaxially grown heterostructures are likely to show little stress at the interface. We investigate under which conditions the scattering region involving $\mathrm{NiMnSb}$ units can exhibit half-metallic properties and ballistic transport in the direction of growth. For a considerable number of bulk half-metallic materials $[2,14]$, the interaction induced NQP states located within the half-metallic gap were proven to significantly reduce the conduction electron spin polarization, while no significant change in the magnetic moment takes place. Here we extend the study on the existence of NQP states at interfaces and investigate their possible impact on steady-state transport.

The paper is organized as follows. Section II gives a brief review of the methodology. The standard equations to compute 
transport properties are presented in Sec. II A. The extension including correlations, in the spirit of dynamical mean field theory (DMFT), is presented in Sec. II B, where we review also the main ideas of the perturbative SPTFLEX-solver [21-23] of DMFT [24-26]. In Sec. III, we discuss the results for the density of states and the transmission in the presence of electronic interactions. Concluding remarks are provided in Sec. IV.

\section{METHODS}

We employ the "two-step" approach presented in our previous paper [27], in which the Landauer transmission probability is calculated within the SMEAGOL nonequilibrium Green's function (NEGF) based electron transport code [28-30]. The package SMEAGOL imports the DFT Hamiltonian from the SIESTA code [31], which uses pseudopotentials and expands the wave functions of valence electrons in the basis of numerical atomic orbitals (NAOs). In the original paper [27], the manybody corrections to the Green's function were evaluated using DMFT [24,26,32] in an exact muffin-tin orbitals (EMTO) based package [33-35], which uses a screened KKR approach [36]. These corrections were then passed to SMEAGOL for the calculation of the transmission matrix. In the present paper, we instead of the EMTO method use the full-potential linearized muffin-tin orbitals (FPLMTO) method, as implemented in the RSPT code $[37,38]$. The FPLMTO method allows us to go beyond shape approximations and treat the full potential, while still keeping a minimal physical basis set. Self-consistent DFT calculations are performed separately in SMEAGOL and in the RSPT code. The many-body self-energy is then evaluated after self-consistency in the RSPT code and passed to the SMEAGOL Green's function to compute the transmission according to the Landauer formalism [39-42].

\section{A. Ballistic transport for electronic systems}

The electronic transport through a device can be addressed in the Landauer formulation [39-42]. According to this approach, the current flow through a device is considered as a transmission process across a finite-size scattering region placed between two semi-infinite leads, connected at infinity to charge reservoirs. The quantity of interest is the conductance, which, within linear response, is given by:

$$
\mathcal{G}=\frac{e^{2}}{h} \frac{1}{\Omega_{\mathrm{BZ}}} \sum_{\sigma=\uparrow, \downarrow} \int_{\mathrm{BZ}} d \mathbf{k}_{\|} T_{\sigma}\left(\mathbf{k}_{\|}, E_{F}\right),
$$

with

$$
\begin{aligned}
& T_{\sigma}\left(\mathbf{k}_{\|}, E\right) \\
& \quad=\operatorname{Tr}\left[\Gamma_{L}^{\sigma}\left(\mathbf{k}_{\|}, E\right) \mathbf{G}^{\sigma \dagger}\left(\mathbf{k}_{\|}, E\right) \Gamma_{R}^{\sigma}\left(\mathbf{k}_{\|}, E\right) \mathbf{G}^{\sigma}\left(\mathbf{k}_{\|}, E\right)\right],
\end{aligned}
$$

where $e$ is the electron charge, $h$ is the Planck constant, and $T_{\sigma}\left(\mathbf{k}_{\|}, E_{F}\right)$ is the spin-dependent transmission probability from one lead to the other for electrons at the Fermi energy with the wave-vector $\mathbf{k}_{\|}$perpendicular to the current flow. The retarded/advanced Green's functions are denoted by $\mathbf{G}^{\sigma}\left(\mathbf{k}_{\|}, E\right) / \mathbf{G}^{\sigma \dagger}\left(\mathbf{k}_{\|}, E\right)$. The integral over $\mathbf{k}_{\|}$extends over the Brillouin zone (BZ) perpendicular to the transport direction, and $\Omega_{\mathrm{BZ}}$ is the area of the BZ. The retarded Green's function
$\mathbf{G}^{\sigma}\left(\mathbf{k}_{\|}, E\right)$ has the following form:

$$
\begin{aligned}
{\left[\mathbf{G}^{\sigma}\left(\mathbf{k}_{\|}, E\right)\right]^{-1}=} & \epsilon^{+} \mathbf{S}\left(\mathbf{k}_{\|}\right)-\mathbf{H}^{\sigma}\left(\mathbf{k}_{\|}\right) \\
& -\Sigma_{L}^{\sigma}\left(\mathbf{k}_{\|}, E\right)-\Sigma_{R}^{\sigma}\left(\mathbf{k}_{\|}, E\right) .
\end{aligned}
$$

All terms presented are matrices, for example, $\left[\mathbf{G}^{\sigma}\left(\mathbf{k}_{\|}, E\right)\right]_{\mu \nu}$, labeled by the global indices $\mu, \nu$ which run through the basis functions at all atomic positions in the scattering region. $\mathbf{S}\left(\mathbf{k}_{\|}\right)$represents the orbital overlap matrix, and the energy shift into the complex plane, $\epsilon^{+}=\lim _{\delta \rightarrow 0^{+}}(E+i \delta)$, has been introduced to respect causality. $\mathbf{H}^{\sigma}\left(\mathbf{k}_{\|}\right)$is the Hamiltonian of the scattering region for spin $\sigma$; the right and left self-energies, $\Sigma_{R}^{\sigma}\left(\mathbf{k}_{\|}, E\right)$ and $\Sigma_{L}^{\sigma}\left(\mathbf{k}_{\|}, E\right)$, describe the energy-, momentum- and spin-dependent hybridization of the scattering region with the right and left leads, respectively [30]. Therefore, $\mathbf{G}^{\sigma}\left(\mathbf{k}_{\|}, E\right)$ is formally the retarded Green's function associated to the effective, non-Hermitian Hamiltonian $\mathbf{H}_{\text {eff }}^{\sigma}\left(\mathbf{k}_{\|}, E\right)=$ $\mathbf{H}^{\sigma}\left(\mathbf{k}_{\|}\right)-\Sigma_{L}^{\sigma}\left(\mathbf{k}_{\|}, E\right)-\Sigma_{R}^{\sigma}\left(\mathbf{k}_{\|}, E\right)$. In Eq. (2), $\Gamma_{L(R)}^{\sigma}\left(\mathbf{k}_{\|}, E\right)=$ $i\left[\Sigma_{L(R)}^{\sigma}\left(\mathbf{k}_{\|}, E\right)-\Sigma_{L(R)}^{\sigma \dagger}\left(\mathbf{k}_{\|}, E\right)\right]$ is the so-called left (right) broadening matrix that accounts for the hybridization-induced broadening of the single-particle energy levels of the scattering region. Importantly, for noninteracting electrons, it has been proven that the Landauer and the Kubo approaches are equivalent [43], so that the linear-response transport properties of a system can be computed with either formalism. The Landauer approach has been systematically applied in conjunction with DFT in order to perform calculations of the conductance of different classes of real nanodevices [44]. In this approach the Kohn-Sham eigenstates are interpreted as single-particle excitations. Although this is only valid approximately, DFT-based transport studies have provided insightful results concerning the role of the band structure in electron transport through layered heterostructures [45-49].

\section{B. Ballistic transport for correlated electrons}

In a multilayer heterostructure the dimensionality of the problem requires a layer-resolved DMFT [24-26] solution for the correlated problem. Therefore, the setup we consider involves a self-consistent calculation for the heterostructure to include the electron-electron interaction beyond the local density approximation (LDA) or the generalized gradient approximation (GGA) explicitly. Accordingly, the retarded Green's function of Eq. (3) has to be modified. We include electron-electron interactions in the form of a multiorbital local Hubbard term $\frac{1}{2} \sum_{i\{m, \sigma\}} U_{m m^{\prime} m^{\prime \prime} m^{\prime \prime \prime}} c_{i m \sigma}^{\dagger} c_{i m^{\prime} \sigma^{\prime}}^{\dagger} c_{i m^{\prime \prime \prime} \sigma^{\prime}} c_{i m^{\prime \prime} \sigma}$ within the interacting region. Here, $c_{i m \sigma}\left(c_{i m \sigma}^{\dagger}\right)$ destroys (creates) an electron with spin $\sigma$ on the orbital $m$ at the site $i$. The Coulomb matrix elements $U_{m m^{\prime} m^{\prime \prime} m^{\prime \prime}}$ are expressed in the standard way [50] in terms of three Kanamori parameters $U, U^{\prime}$, and $J$. The interaction is treated in the framework of DMFT [26,32,51], with a spin-polarized T-matrix fluctuation exchange (SPTF) type of impurity solver [21,52] implemented within the FPLMTO basis set $[37,38]$. The SPTF approximation is a multiband spin-polarized generalization of the fluctuation exchange approximation (FLEX) [53,54]. In the context of lattice models it describes the interaction of quasiparticles with collective modes. From a formal point of view, it is a perturbative expansion of the self-energy in powers 
of $U$, with a resummation of specific classes of diagrams, such as ring and ladder diagrams. The expansion remains reliable as long as the strength of the interaction $U$ is smaller than the bandwidth of the material. This is a valid approach for $\mathrm{NiMnSb}$ as its bandwidth is about $\approx 8 \mathrm{eV}$, and the relevant values for the local Coulomb parameter are in the range of $U \approx 2 \ldots 3 \mathrm{eV}$ [14]. Justifications, further developments, and details of this scheme can be found in Ref. [21].

For the case of half-metallic ferromagnets it was demonstrated [2] by model as well as realistic electronic structure calculations that many-body effects are crucial for half-metals: They produce states with tails that cross the Fermi level so that the gap is closed and half-metallicity is lost [14-17,27,55]. The origin of these many-body NQP states is connected with "spin-polaron" processes: The spin-down low-energy electron excitations, which are forbidden for the HMF in the one-particle picture, turn out to be allowed as superpositions of spin-up electron excitations and virtual magnons [2,56]. Spinpolaron processes are described within the SPTF approach by the fluctuation potential matrix $W^{\sigma \sigma^{\prime}}\left(i \omega_{n}\right)$, with $\sigma= \pm$, defined as follows [54]:

$$
\hat{W}(i \omega)=\left(\begin{array}{ll}
W^{++}\left(i \omega_{n}\right) & W^{+-}\left(i \omega_{n}\right) \\
W^{-+}\left(i \omega_{n}\right) & W^{--}\left(i \omega_{n}\right)
\end{array}\right) .
$$

The essential feature here is that the potential (4) is a complex energy-dependent matrix in spin space with offdiagonal elements:

$$
W^{\sigma,-\sigma}\left(i \omega_{n}\right)=U^{\mathbf{m}}\left(\chi^{\sigma,-\sigma}\left(i \omega_{n}\right)-\chi_{0}^{\sigma,-\sigma}\left(i \omega_{n}\right)\right) U^{\mathbf{m}},
$$

where $U^{\mathbf{m}}$ represents the bare vertex matrix corresponding to the transverse magnetic channel, m. The susceptibility $\chi^{\sigma,-\sigma}\left(i \omega_{n}\right)$ is the effective transverse susceptibility matrix, and $\chi_{0}^{\sigma,-\sigma}\left(i \omega_{n}\right)$ is the bare transverse susceptibility [54]. The Matsubara frequencies are the complex energies $i \omega_{n}=$ $i(2 n+1) \pi T$, where $n=0,1,2, \ldots$ and $T$ is the temperature $[53,54]$. The local Green's functions as well as the electronic self-energies are spin diagonal for collinear magnetic configurations. In this approximation the electronic self-energy is calculated in terms of the effective interactions in various channels. The particle-particle contribution to the self-energy was combined with the Hartree-Fock and the second-order contributions [54]. To ensure a physical transparent description the combined particle-particle self-energy is presented by Hartree, $\Sigma^{(T H)}\left(i \omega_{n}\right)$, and Fock, $\Sigma^{(T F)}\left(i \omega_{n}\right)$, type contributions: $\Sigma\left(i \omega_{n}\right)=\Sigma^{(T H)}\left(i \omega_{n}\right)+\Sigma^{(T F)}\left(i \omega_{n}\right)+\Sigma^{(\mathrm{ph})}\left(i \omega_{n}\right)$, where the particle-hole contribution $\Sigma^{(\mathrm{ph})}$ reads:

$$
\Sigma_{12 \sigma}^{(\mathrm{ph})}\left(i \omega_{n}\right)=\sum_{34 \sigma^{\prime}} W_{1342}^{\sigma \sigma^{\prime}}\left(i \omega_{n}\right) G_{34}^{\sigma^{\prime}}\left(i \omega_{n}\right) .
$$

A Padé [57,58] analytical continuation is employed to map the self-energies from the Matsubara frequencies onto real energies, as required in the transmission calculation. Since the static contribution from correlations is already included in the exchange-correlation potential of DFT, the so-called "doublecounted" terms must be subtracted. In other words, those parts of the DFT expression for the total energy that correspond to the interaction included in the Hubbard Hamiltonian has to be subtracted. To achieve this, we replace $\Sigma_{\sigma}(E)$ with $\Sigma_{\sigma}(E)-\Sigma_{\sigma}(0)$ [59] in all equations of the DMFT procedure
[51]. Physically, this is related to the fact that DMFT only adds dynamical correlations to the LSDA/GGA result. For this reason, it is believed that this kind of double-counting subtraction is more appropriate for a DMFT treatment of metals than the alternative static Hartree-Fock (HF) subtraction [60].

The analytically continued self-energy (obtained in the DMFT), within the FPLMTO basis set, is transferred into the multiple-zeta basis of SIESTA according to the basis transformation presented in Ref. [27]. With the corresponding self-energy we compute the interacting Green's function and use the latter in the expression for the transmission:

$$
\begin{aligned}
\mathbf{G}_{\mathrm{DMFT}}^{\sigma}\left(\mathbf{k}_{\|}, E\right)=\left[\epsilon^{+}\right. & \mathbf{S}\left(\mathbf{k}_{\|}\right)-\mathbf{H}^{\sigma}\left(\mathbf{k}_{\|}\right)-\Sigma_{L}^{\sigma}\left(\mathbf{k}_{\|}, E\right) \\
- & \left.\Sigma_{R}^{\sigma}\left(\mathbf{k}_{\|}, E\right)-\Sigma_{\mathrm{DMFT}}^{\sigma}(E)\right]^{-1}, \\
T_{\mathrm{DMFT}}^{\sigma}\left(\mathbf{k}_{\|}, E\right)= & \operatorname{Tr}\left[\Gamma_{L}^{\sigma}\left(\mathbf{k}_{\|}, E\right) \mathbf{G}_{\mathrm{DMFT}}^{\sigma \dagger}\left(\mathbf{k}_{\|}, E\right)\right. \\
& \left.\times \Gamma_{R}^{\sigma}\left(\mathbf{k}_{\|}, E\right) \mathbf{G}_{\mathrm{DMFT}}^{\sigma}\left(\mathbf{k}_{\|}, E\right)\right] .
\end{aligned}
$$

The self-energy acts as a spin- and energy-dependent potential whose imaginary part produces a broadening of the single-particle states due to the finite electron-electron scattering lifetime. Note that this is an approximation, since it neglects vertex corrections due to in-scattering processes $[61,62]$, which in general increase the conductivity. Since we are not performing fully self-consistent transmission calculations our $\Gamma_{L / R}^{\sigma}\left(\mathbf{k}_{\|}, E\right)$ remain at the LDA/GGA level. We are not aware of any method that allows for fully self-consistent calculations of $\Gamma_{L / R}^{\sigma}\left(\mathbf{k}_{\|}, E\right)$ for realistic materials.

\section{Computational details}

Bulk NiMnSb crystallizes in the face-centered cubic (fcc) structure with three atoms per unit cell, with positions Ni $(0,0,0), \mathrm{Mn}\left(\frac{1}{4}, \frac{1}{4}, \frac{1}{4}\right)$, and $\mathrm{Sb}\left(\frac{3}{4}, \frac{3}{4}, \frac{3}{4}\right)$. In the FPLMTO calculations, the radii of the nonoverlapping muffin-tin spheres were set to 2.02 a.u. (Ni), 1.99 a.u. (Mn), 2.21 a.u. (Sb), and 2.38 a.u. ( $\mathrm{Au})$ respectively. For $\mathrm{Ni}$ and $\mathrm{Mn}$ the $3 s, 3 p, 4 s$, and $3 d$ electrons, and for $\mathrm{Sb}$ the $5 s, 5 p$, and $4 d$ electrons, were considered to be valence electrons. For the Au leads $6 s$ and $5 d$ electrons where treated as valence states. For the bulk case a k-point mesh of size $32 \times 32 \times 32$ was employed. The angular momentum cutoff for the charge density was chosen as $l_{\max }=8$. Three kinetic energy tails corresponding to 0.3 , -2.3 , and -1.5 Ry were employed. The exchange-correlation potential was approximated using the Perdew and Wang parametrization for the LSDA [63]. We confirm that no change in the magnitude of the minority spin gap is obtained using the GGA functional [64] and no significant difference between the DOS spectra can be seen, while the position of the Fermi level is changing within $0.1 \%$. By direct computation [65], spin-orbit effects were found to be negligible for the quantities of interest, and hence we only kept the scalar-relativistic terms in our calculations. The Matsubara sums were truncated after 1024 frequencies, and the temperature was set to $316 \mathrm{~K}$ (corresponding to $2 \mathrm{mRy}$ ).

The details of the transport calculations within the Landauer formalism are briefly mentioned in the following. The basis set used in the SIESTA and SMEAGOL calculations is of "double-zeta with polarization" (DZP) quality. The "energy shift" parameter which allows us to control the extent of basis functions on 
different atoms is taken as $300 \mathrm{meV}$; this choice resulted in basis functions with a maximum extent of $6.1 a_{0}(\mathrm{Au}), 6.0 a_{0}$ $(\mathrm{Ni}), 6.4 a_{0}(\mathrm{Mn})$, and $4.9 a_{0}(\mathrm{Sb})$ where $a_{0}$ is the Bohr radius. As the results of the electronic structure are independent on the choice of exchange correlation functional, we used further the PBE-GGA [66] implementation; however, as discussed in Sec. III A, no relaxation is needed.

\section{RESULTS}

This section presents the results for the electronic structure of bulk $\mathrm{NiMnSb}$ and for different terminations of $\mathrm{NiMnSb}(001)$ interfaces. We compare with previous studies at the level of DFT (LDA/GGA), and then include electronic correlations using DMFT. The results for the $\mathrm{NiMnSb}(001)$ interfaces are compared with the $\mathrm{NiMnSb}(001)$ interface to $\mathrm{Au}$; for the latter the corresponding transmission coefficients are discussed.

\section{A. Electronic structure of bulk NiMnSb and $\mathrm{NiMnSb}(001)$ interfaces with vacuum or Au}

\section{General results concerning bulk and surface studies}

Several calculations using DFT methods are available for bulk NiMnSb [1,2,64] and its surface states [10,67]. According to these results, the bulk minority spin indirect gap is formed between the $\Gamma$ and $X$ points of the Brillouin zone corresponding to the fcc unit cell. The minority spin bands consist of completely occupied $\mathrm{Sb} p$ states $[1,2]$, while the bonding and antibonding $d$ hybrids of the $\mathrm{Mn}$ and $\mathrm{Ni}$ atoms are separated by the half-metallic gap. The bonding states have most of their weight at the $\mathrm{Ni}$ atom, and the antibonding states at the Mn atom, leading to large localized spin moments at the $\mathrm{Mn}$ atoms [68]. The total spin moment follows the Slater-Pauling behavior as shown in Ref. [64], being exactly $4 \mu_{B}$. This value is mainly determined by the ferromagnetic alignment of the large Mn spin moment $\left(3.72 \mu_{B}\right)$ and a small Ni contribution $\left(0.28 \mu_{B}\right)$. Including spin-orbit coupling leads to a partially filled minority spin gap [65]. However the changes are negligible: The majority spin DOS around the Fermi level changes only marginally, and the material remains essentially half-metallic with a polarization of the DOS of about 99\% [65]. DMFT calculations, on the contrary, show the presence of NQP states, just above the Fermi level [14].

Terminations of $\mathrm{NiMnSb}(001)$ surfaces are possible either with a $\mathrm{Ni}$ or a MnSb layer. These interfaces were studied previously [67,69], and great attention has been given to relaxation effects. The results for the first two interface layers can be briefly summarized as follows. (i) In the case of the $\mathrm{Ni}$ termination, almost no buckling or relaxation of the $\mathrm{MnSb}$ subsurface layer was observed, while the distance between the top Ni layer and the subsurface layer was reduced by around $10 \%$. (ii) In the case of the $\mathrm{MnSb}$ termination, the $\mathrm{Mn}$ atom at the surface layer moves inwards and the Sb atom outwards: The distance between the Mn surface atom and the Ni subsurface layer is contracted by $3.5 \%$, and the distance between the $\mathrm{Sb}$ surface atom and the Ni subsurface layer is expanded by $7.3 \%$.

Relaxation effects on the DOS have also been discussed, however, no significant relaxation effects are seen [7-9,70]. Strongly localized surface states belonging to the top surface layer contribute to the in-gap states, while no states from the subsurface layer are present $[67,69]$. The Ni-terminated surface states are localized to particular surface layers and lead to flat dispersions. Accordingly, the DOS of these surface states is much more pronounced compared to those of $\mathrm{MnSb}$ terminated surface states and effectively destroy the minority spin gap $[67,69]$.

\section{Electronic structure of bulk NiMnSb}

In Fig. 1 we present the LSDA and LSDA+DMFT results for the total DOS of bulk NiMnSb (left) and of slab
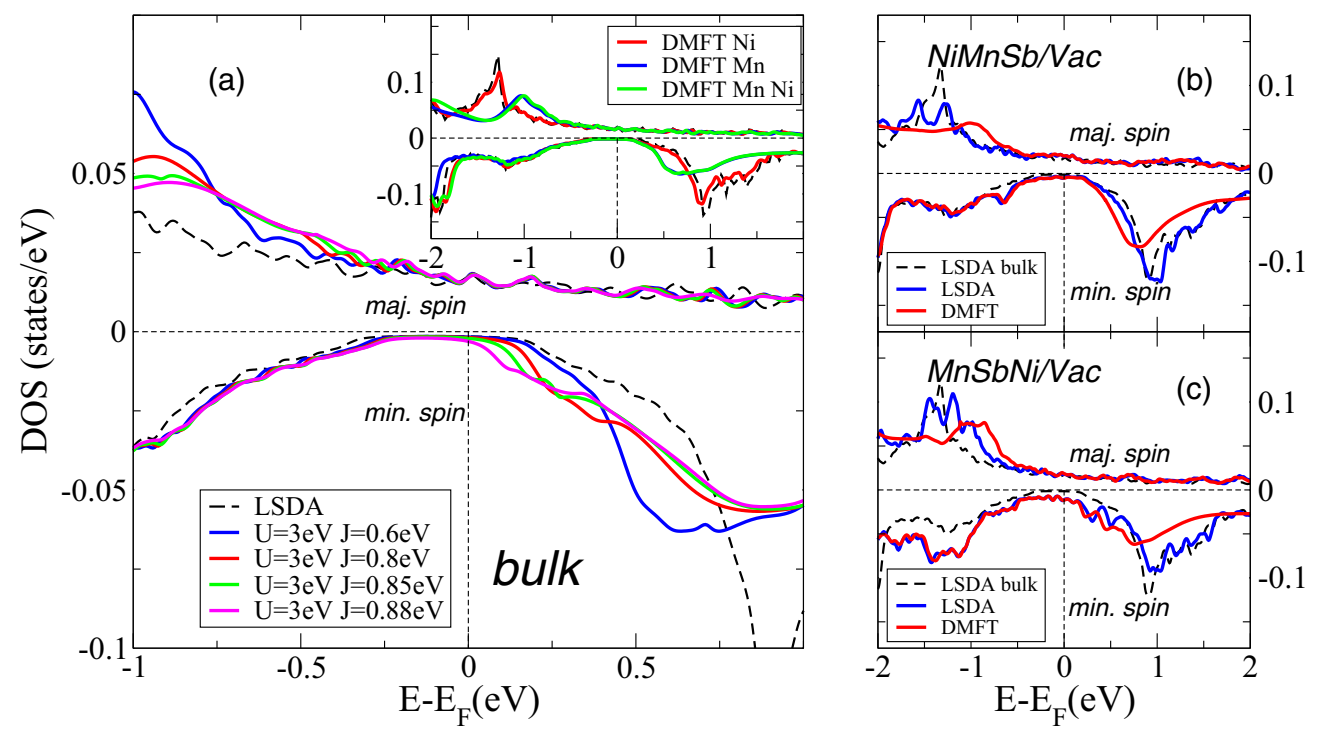

FIG. 1. [Left panel, (a)] Total DOS for bulk NiMnSb. The black dashed lines indicate the LSDA results. Solid lines represent LSDA+DMFT results including the Hubbard interaction $(U=3 \mathrm{eV})$ and various Hund's exchange couplings (only Mn treated as correlated). The inset shows the DOS for $U=3 \mathrm{eV}$ and $J=0.6 \mathrm{eV}$. The legend indicates which species are treated as correlated, respectively. (Right panel) Total DOS for the (NiMnSb)/Vac interface with two possible (001) surface terminations. (b) MnSb-termination, NiMnSb/Vac, (c) Ni-termination, $\mathrm{MnSbNi} / \mathrm{Vac}$. Solid lines: LSDA (blue) and DMFT (red). In the DMFT calculations we again used $U=3 \mathrm{eV}$ and $J=0.6 \mathrm{eV}$. 
$\mathrm{NiMnSb} / \mathrm{Vac}$ (right). Figure 1, left panel, represents the DOS in the energy window $E_{F} \pm 1 \mathrm{eV}$ around the half-metallic gap. In its inset the total DOS is presented in the larger energy range $E_{F} \pm 2 \mathrm{eV}$. To facilitate the comparison, the same energy range is used for the total DOS of the slab calculations (Fig. 1, right panel).

The LSDA results for bulk NiMnSb correspond to the case in which no $U$ and $J$ corrections are applied. A minority spin gap of $\approx 0.5 \mathrm{eV}$ is visible. We call the energy range where the minority spin DOS develops a gap in the LSDA calculation the gap region. The computed gap is in good agreement with the experiment of Kirillova et al. [71], who analyzed infrared spectra and estimated the gap to be $\approx 0.4 \mathrm{eV}$. Previously published $[1,2,64,72]$ band structure calculations report band gaps similar to our result. The minority spin gap is formed within the $d$-band manifold between the bonding $\left(t_{2 g}\right)$ and antibonding $\left(e_{g}\right)$ states. Above the Fermi level, Mn $d$ orbitals dominate the gap edge.

In order to study the electron-electron correlation effects and their influence on the minority spin gap, we performed calculations with different values of the local Coulomb interaction and exchange parameters up to $U=3 \mathrm{eV}$ and $J=0.88 \mathrm{eV}$. When the $\mathrm{Mn}$ sites are treated as correlated, we observe significant changes in the DOS (see Fig. 1): Peaks are shifted and simultaneously the gap is filled just above the Fermi level. These results are in agreement with previous LSDA+DMFT calculations [14]. The origin of the in-gap states is connected with "spin-polaron" processes [2,14,73-75]. The density of NQP states vanishes at $E_{F}$ at zero temperature and increases on the energy scale of the order of a characteristic magnon frequency [2,14,73-75]. This has been calculated from first principles for semi-Heuslers $[14,15]$, Heuslers [16,76], zinc-blend half-metals [55,77], and heterostructures [78,79].

For bulk NiMnSb we have studied the formation of the NQP state as a function of the interaction parameters. In particular, we observe that the Hund's coupling $J$, the intra-atomic exchange energy, crucially influences the DOS. Increasing the value of $J$ in the range from $0.6 \mathrm{eV}$ up to about $0.88 \mathrm{eV}$, the NQP state (broad shoulder) is shifted towards the Fermi level. Such behavior can be captured within a $s-d$ type model for the electron-magnon interaction $[2,74,75]$ and was shown to be valid also for the Hubbard model within the DMFT approximation [14]. In the $s-d$ model case, the corresponding change, $\delta N_{\sigma}(E)$, with respect to the noninteracting halfmetallic DOS has the form:

$$
\begin{aligned}
\delta N^{\sigma}(E) & =N^{\sigma}(E)-N_{0}^{\sigma}(E) \\
& =-\sum_{\mathbf{k}}\left[\Re \Sigma^{\sigma}(\mathbf{k}, E) \delta^{\prime}\left(E-\epsilon_{\mathbf{k} \sigma}\right)-\frac{1}{\pi} \frac{\Im \Sigma^{\sigma}(\mathbf{k}, E)}{E-\epsilon_{\mathbf{k} \sigma}}\right] .
\end{aligned}
$$

The first term of Eq. (9), proportional to the real part of the self-energy, causes the shift of the quasiparticle energies. The second term arising from the branch cut of the self-energy describes the incoherent, NQP contribution and is proportional to the imaginary part of the self-energy. Using the local approximation of DMFT it amounts to replace $\Sigma^{\sigma}(\mathbf{k}, E)$ with $\Sigma_{\text {DMFT }}^{\sigma}(E)$. All these effects are visible in Fig. 1, hence our numerical results confirm that the formation of NQP states, in the multiorbital Hubbard model for the HMF NiMnSb, is a consequence of the low-energy electron-magnon physics captured by the $s-d$ model.

In the inset we show a comparison between the LSDA+DMFT results when both, or only one of the $d$-electron subsystems, are treated as correlated. Adding the Hubbard corrections only to $\mathrm{Ni}$, we find only minor changes compared to the LSDA results. Considering both $\mathrm{Mn}$ and $\mathrm{Ni}$ as correlated, the results do not differ much from the case in which only $\mathrm{Mn}$ is treated as correlated. Since correlation effects originating from the Ni site affect the DOS only slightly in the energy range close to $E_{F}$, we treat $\mathrm{Ni}$ as uncorrelated in the following surface and interface calculations.

\section{Electronic structure at the NiMnSb/vacuum interface}

On the right hand side of Fig. 1 we present the total DOS for supercells containing two NiMnSb units and eight layers of vacuum. The two possible (001) surface terminations are shown. Results for the MnSb termination (top part) and the Ni termination (bottom part) on the surfaces of the slab are displayed. The overall shape of the LSDA-DOS is similar to the bulk one for both surface terminations, except in the gap region: We find that the gap in the minority spin DOS disappears and instead in-gap localized surface states are formed. The in-gap states for Ni-terminated surfaces are more intense than those for MnSb-terminated surfaces. Our LSDA results agree with previous calculations [67,69]. Independent of the surface termination, the peak in the bulk DOS (around $E-E_{F} \approx-1.5 \mathrm{eV}$ ) of hybrid $\mathrm{Ni}$ and $\mathrm{Mn}$ bonding states of $e_{g}$ character is reduced in magnitude and splits. The splitting of this bulk peak is a consequence of the symmetry reduction from cubic to tetragonal. The weight is redistributed and the overall magnitude of the DOS decreases.

The LSDA+DMFT results for the DOS follow the general trend of the corresponding results for the bulk. The in-gap surface states are already present at the LSDA level. They are located in the same energy range as the NQP states, hence the NQP peak is not discernible in the DOS. The only remaining visible correlation effect is the shift of the one-particle states. In comparison with the LSDA spectra the unoccupied minority spin states and the occupied majority spin states shift towards the Fermi level.

\section{Electronic structure at the NiMnSb/Au interface}

Next we consider the contact geometry as shown in Fig. 2. The geometry is similar to the surface structures discussed above but with the vacuum replaced by metallic gold leads. For the NiMnSb/Au interface the contact is taking place between the gold layer and one of the two possible layer terminations: The Ni-terminated structure is shown as the left part, and the MnSb-terminated structure is shown as the right part of Fig. 2, respectively.

In the upper and lower parts of Fig. 3 we present the majority and the minority total DOS, respectively, for the case in which two NiMnSb units in the interacting region are considered. With blue and red lines we denote, respectively, the LSDA and the DMFT results. In the left and right panels the results for the $\mathrm{Ni}$ - and $\mathrm{MnSb}$-terminated interfaces are 

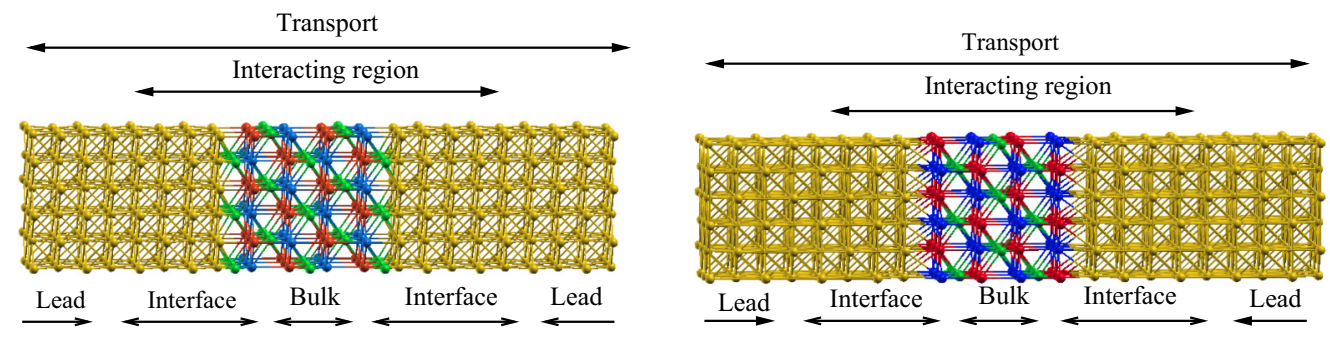

FIG. 2. Schematic representation of the supercell used in the calculations. (Left) Ni-terminated (001) interface. (Right) MnSb-terminated interface. We indicate the regions where the electronic structure is assumed similar to that of the bulk as well as the size of the cells used in transport and DFT calculations, respectively. Atomic color code: Ni (green), Mn (blue), Sb (red), Au (yellow).

given. For comparison we have plotted in Fig. 3 also the bulk NiMnSb DOS (dashed lines).

For both interfaces one clearly sees that states appear inside the minority spin gap region, both in LSDA and LSDA+DMFT calculations. These states are most likely localized near the interface region, similarly to the case of the $\mathrm{NiMnSb}(001)$ surface [69]. However, because of the presence of Au layers, a stronger weight of the in-gap states is obtained. Note that significant differences between correlated and noncorrelated calculations are not seen in the close vicinity of $E_{F}$ for both spin channels. For the Ni termination the minority spin gap is populated with an almost constant DOS [Fig. 3(a), left panel]. For the MnSb termination the minority spin DOS just above the Fermi level reaches a maximum at about $0.1 \mathrm{eV}$ [Fig. 3(b), right panel]. No such maximum in the DOS is present in the vicinity of $E_{F}$ for the interface calculation with vacuum as compared to Fig. 1(b). The origin of this maximum in the minority spin DOS, just above the Fermi level, can be attributed to the hybridization between the $\mathrm{Au} s / p$ and the $\mathrm{Sb} s / p$ orbitals of the $\mathrm{Au} / \mathrm{MnSb}$ interface. For $\mathrm{Ni}$ termination such hybridization cannot take place, therefore the constant DOS in the minority spin channel [Fig. 3(a), left panel] represents in fact the interface Ni states.

Correlation effects are visible only outside the gap region for both spin channels. For both terminations, changes in the DOS occur below $E_{F}$ for the majority spin electrons and above
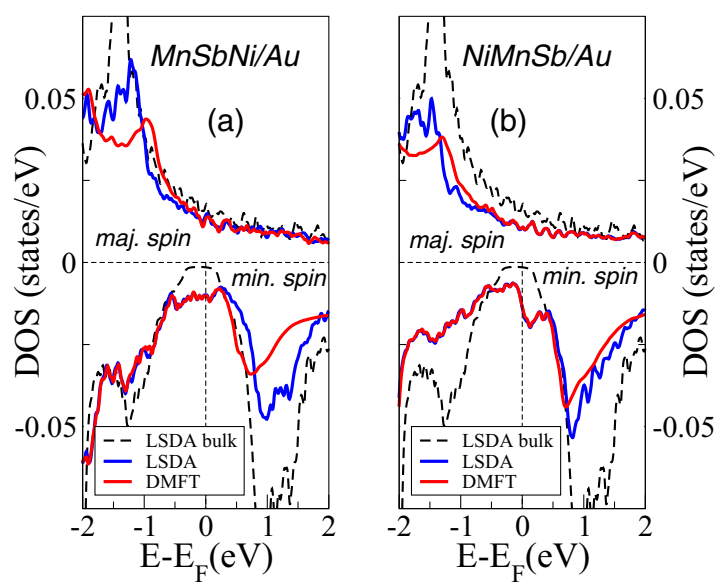

FIG. 3. Total DOS for the Au-(NiMnSb) $)_{2}$-Au slab. (a) Ni termination, (b) MnSb termination. The solid-blue line indicates the LSDA DOS and the solid-red line indicates the LSDA+DMFT DOS. The LSDA+DMFT results were obtained for $U=3 \mathrm{eV}$ and $J=0.6 \mathrm{eV}$.
$E_{F}$ for the minority spin electrons. These changes represent correlation-induced spectral weight shifts, resulting from the negative slope of the real part of the self-energy. The signature of many-body features in the spectral function of the HMFs under consideration is the existence of NQP states. These are generic many-body features present in all ferromagnetic materials, however, in half-metals their weight and position is visible because of the existence of the gap. In bulk NiMnSb the NQP states are apparent in Fig. 1; their weight depends significantly on the strength of the exchange coupling $J$, and they inherit the $d$-orbital character of the Mn atoms [2,14]. The nonquasiparticle contributions to the DOS originates from the imaginary part of the minority spin self-energy $[2,74,75]$, which produces a branch cut in the corresponding Green's function. A similar result exists for the surface states [73] and can be generalized for arbitrary inhomogeneous situations [75], thus it applies also to interfaces. In order for the NQP states to become visible in the spectral function (DOS), interface half-metallicity is required, with the gap formed between correlated orbitals. In the present case of the $\mathrm{NiMnSb} / \mathrm{Au}$ interface, the half-metallic character is lost because of the interface hybridization in the case of $\mathrm{MnSb}$ termination or because of extended interface $\mathrm{Ni}$ states in the Ni-terminated setup. Consequently, NQP states cannot be clearly discriminated in the DOS, since they are overlayed by the interface states.

In situations where the gap region in the minority spin channel is filled by surface states, the minority spin tunneling process is mediated by those states only [75]. Although the NQP states are overlayed in the DOS, their presence can in principle be revealed through the tunneling current due to hybridization of bulk states with surface or interface states [73,75].

\section{B. Transport properties: the Au-NiMnSb-Au heterostructure}

Within the standard approach for ballistic transport calculations, the system is partitioned into two semi-infinite parts (the leads) and the scattering region. Generally speaking, the transmission probability is essentially determined by the scattering matrix connecting the states from the left lead to those in the right lead. The structural setup consists of two gold leads in the $\mathrm{Au}(001)$ orientation that sandwich atomic planes in the pattern $\mathrm{AB}-\mathrm{AB}-\mathrm{AB}(\mathrm{A}=\mathrm{Ni}, \mathrm{B}=\mathrm{MnSb})$ along the transport direction. The Au leads structure is built using the experimental value for the bulk lattice parameter $(4.08 \AA)$. The NiMnSb units are built on top of this structure and oriented accordingly with the two possible terminations 

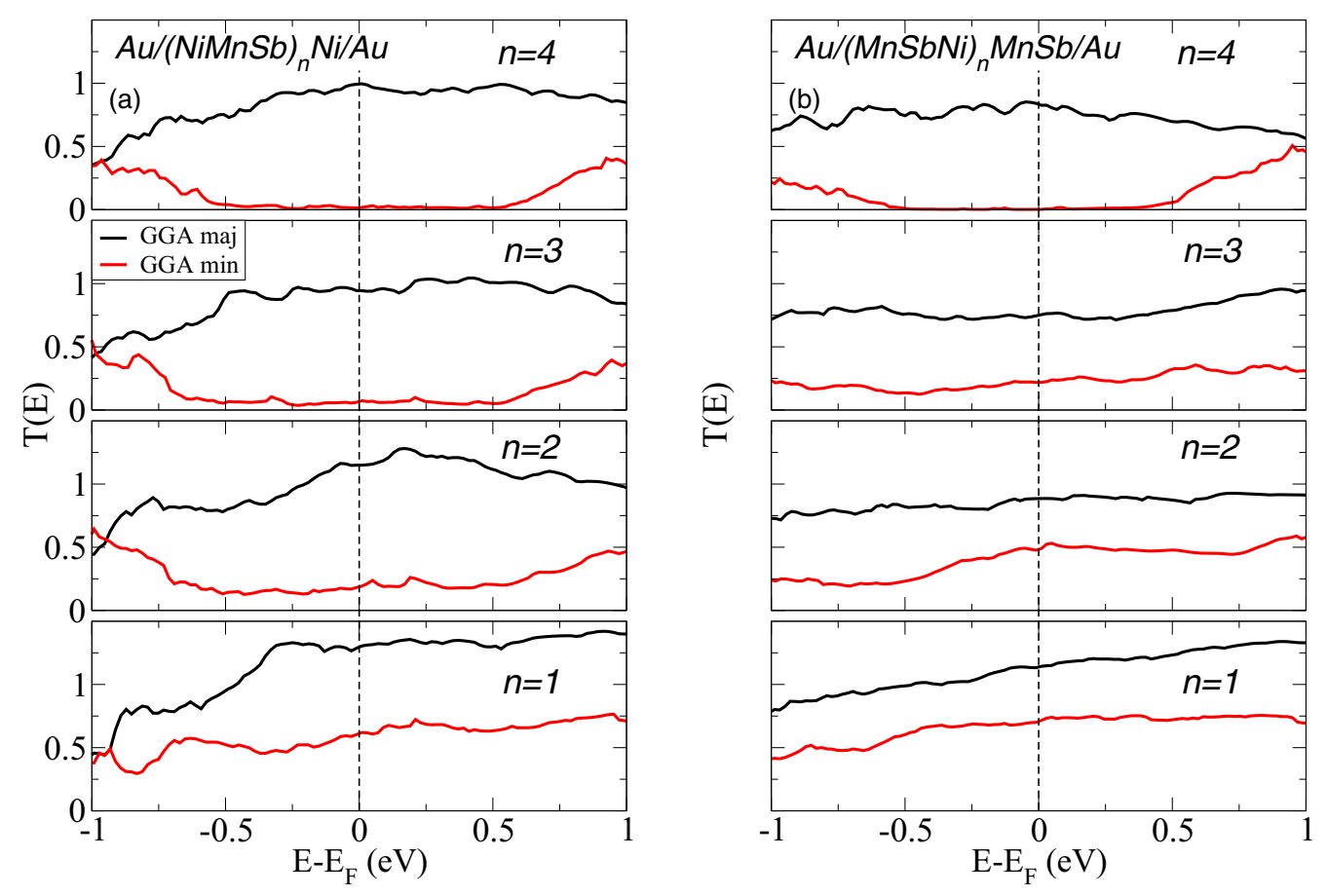

FIG. 4. DFT (GGA) spin-resolved transmission versus the number $n$ of (NiMnSb) ${ }_{n}$ units: (a) Ni-terminated interfaces and (b) MnSbterminated interfaces. The black solid lines represent the majority and the red solid lines the minority spin transmission, respectively.

discussed above (see Fig. 2). The region where scattering occurs (the interacting region) contains a few layers of the two leads as well as sequences of $\mathrm{Ni}$ and $\mathrm{MnSb}$ layers as indicated in Fig. 2. The electronic structure of the NiMnSb units within the interacting region is different from that of the bulk, due to the presence of the interface (see Sec. III A). For thick layers (e.g., including three or more units of $\mathrm{NiMnSb}$ ) we expect to find the electronic structure of bulk NiMnSb in the center of the interaction zone. Therefore, for such thick layers, the scattering properties across the whole structure should reflect the electronic structure of the bulk, as confirmed below.

\section{Transmission within $G G A$}

First we analyze the transmission, with focus on the changes brought by the presence of the interfaces and hence the electronic structure. The spin-resolved transmission probability, $T_{\sigma}(E)$, is obtained from the k-dependent transmission by integrating over all $\mathbf{k}_{\|}$points, so that $T_{\sigma}(E)=$ $\frac{1}{\Omega_{\mathrm{BZ}}} \int_{\mathrm{BZ}} d \mathbf{k}_{\|} T_{\sigma}\left(\mathbf{k}_{\|}, E\right)$. The $\left(\mathbf{k}_{\|}, E\right)$-dependent transmission, Eq. (2) or (8), is obtained from the matrix product of the hybridizations $\Gamma_{L / R}^{\sigma}\left(\mathbf{k}_{\|}, E\right)$ and the retarded/advanced Green's functions $\mathbf{G}^{\sigma}\left(\mathbf{k}_{\|}, E\right) / \mathbf{G}^{\sigma \dagger}\left(\mathbf{k}_{\|}, E\right)$. While the Green's functions encode the electronic structure of the interacting region, the hybridization functions contain the information about the hopping of electrons into and out of the interacting region.

In Fig. 4 we display the total spin-resolved transmission probability computed within the GGA. It can be immediately seen that the transmission is strongly spin-dependent. For more than three units, the spin polarization at $E_{F}$ is above $90 \%$ for both terminations. Thus, for both the Ni- and the MnSbterminated structure, the "bulk-like" behavior (i.e., "halfmetallic" transmission) is present already for four $\mathrm{NiMnSb}$ units. For smaller $n$, we argue that a direct lead-to-lead conduction channel forms which leads to nonzero transmission for the minority spin electrons. The transmission in the majority spin channel (solid black lines) is slightly smaller for the MnSb-terminated structure in comparison with the Ni-terminated structure.

\section{Transmission within GGA+DMFT}

Second we analyze the transmission in the view of the modifications brought by the presence of electronic correlations. The GGA+DMFT transmissions for $\mathrm{Ni}$ - and MnSb-terminated structures are shown in Fig. 5. Overall the GGA+DMFT and GGA transmissions have a similar energy dependence, however, the magnitude is found to be reduced due to electronic correlations.

For the Ni-terminated structure (left column of Fig. 5) correlation-induced changes are visible above the Fermi level in both spin channels. For the MnSb-terminated structure, the modifications are spin selective. For the majority spin (black line, Fig. 5, right column) a more significant reduction in transmission is seen below $E_{F}$. This is a consequence of the (larger) smaller weight of the imaginary part of the $\mathrm{Mn}$ self-energy (below) above $E_{F}$. The opposite effect is found for the minority spin electrons (red line). In this spin channel the imaginary part of the Mn self-energy has a (smaller) larger weight (below) above the Fermi energy.

The depletion in the transmission is determined by the decrease in the coherence of the scattered wave function across the heterostructure. The inverse of the finite imaginary part of the self-energy corresponds to a finite lifetime of the quasiparticle, hence broadening the spectral function and the DOS. This is valid for all layers in the scattering region that are subject to electronic correlations. We find that the correlation-induced changes of the transmission are almost 

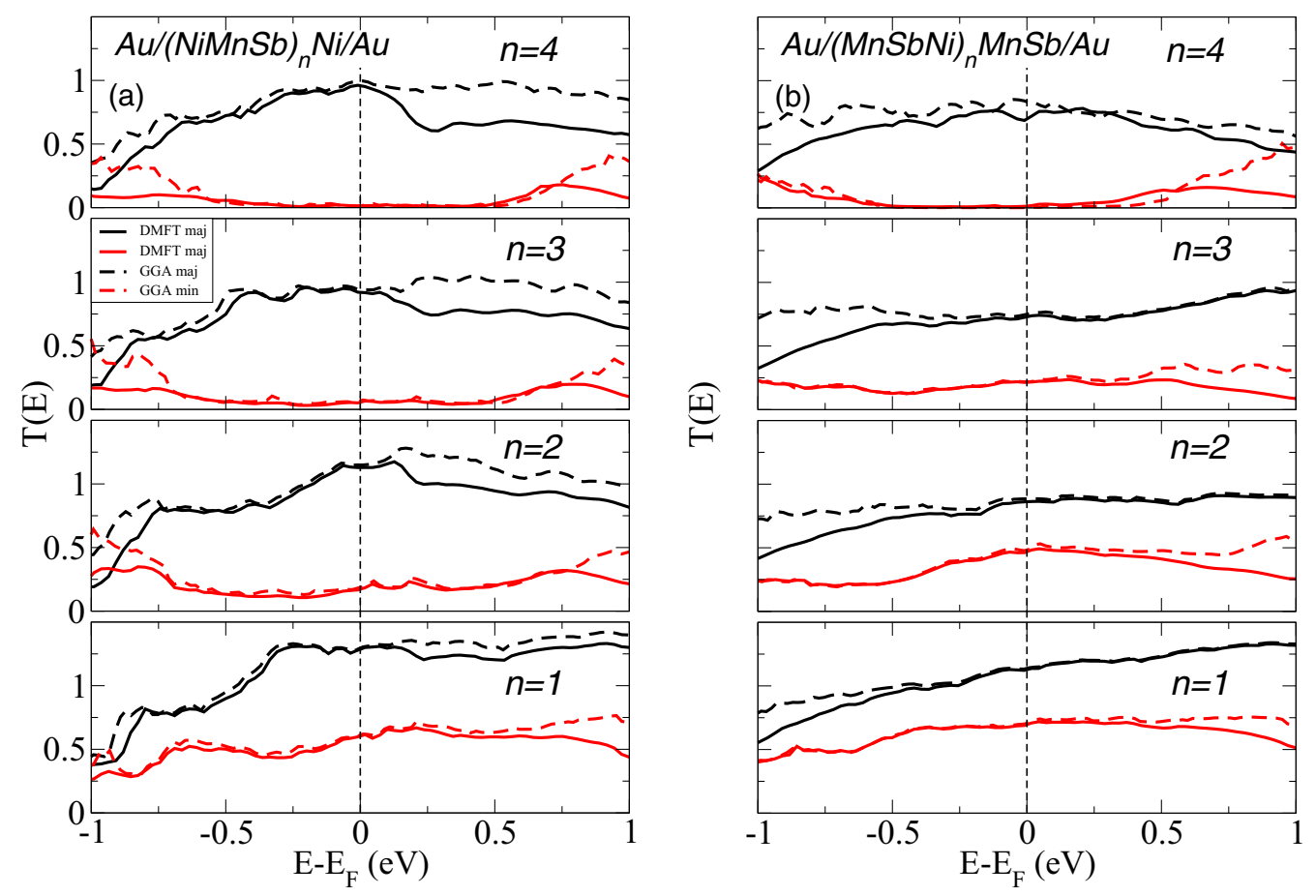

FIG. 5. Spin-resolved transmissions. The dashed lines represent the GGA and the solid lines the GGA+DMFT results. The black colored lines denote the majority spin transmission and the red colored lines the minority spin transmission: transmission through the (a) Ni-terminated structure and (b) through the MnSb-terminated structure.

independent of $n$. For $n<3$ the tunneling is influenced considerably by lead-to-lead direct tunneling, and signatures of the electronic structure of NiMnSb are not clearly visible. For $n=4$, a significant spin polarization of the transmission sets in, in particular, for the MnSb-terminated case, indicating the relevance of half-metallicity in the scattering region. Away from the Fermi level a hump is formed at $0.4 \ldots 0.6 \mathrm{eV}$ in the minority spin channel.

The surface and interface electronic structure calculations in Sec. III A 2 show that the minority spin gap is filled by surface states, which are more important than many-body effects (the NQP features in Fig. 1). A more quantitative analysis can be based on the direct comparison of the DOS with the transmission near $E_{F}$. In the upper part of Fig. 6, we show the DOS for the heterostructures with four units of $\mathrm{NiMnSb}$ in the scattering region, Ni-terminated (left) and MnSb-terminated (right). In the lower part of Fig. 6, the transmissions are presented for the corresponding structures. We focus on a narrow energy range around the Fermi level, $E_{F} \pm 0.4 \mathrm{eV}$. For the Ni-terminated structure, no correlationinduced change in the DOS is seen in the range -0.4 to $0.2 \mathrm{eV}$. On the other hand, the transmission (lower part) is reduced in the entire energy range. For the MnSb-termination, we find no significant change in the DOS in the $E_{F} \pm 0.4 \mathrm{eV}$ energy range. However, the transmission is clearly enhanced by electronic correlations. This might be understood as tunneling assisted by quasiparticle states or by the nonquasiparticle states in the scattering region [75].

The modification of the transmission probability caused by the finite real part of the self-energy can be interpreted as a renormalization of the one-particle states within the scattering region. A second mechanism that modifies the transmission amplitude is given by the incoherent part of the minority spin self-energy caused by hybridization of surface states with NQP states.

In order to resolve the true nature of the changes in the minority spin transmission, one should investigate the temperature and bias dependence of the tunneling current. It has been shown previously that the quasiparticle contribution to the tunneling current can be even more pronounced in comparison to the NQP peak in the DOS [75]. It is worth emphasizing that in the Landauer approach the many-body equilibrium self-energy is used to compute the transmission in a first step, which is then used to determine the tunneling current in a second step. This implies that the bias and temperature dependence of the tunneling current is solely due to the Fermi-Dirac distribution which contains these parameters. Hence the Landauer formalism explicitly excludes genuine correlation effects on the bias dependence of the tunneling current by construction. We believe that the hump around $0.4 \ldots 0.6 \mathrm{eV}$ (seen in Fig. 5) in the minority spin transmission can be considered a precursor of the bias dependence of the tunneling current. In order to address the implications of this hump in the minority spin transmission in a more rigorous way, an extension of the present transport formalism to a full charge self-consistent calculation, employing the NEGF including many-body corrections on the level of DMFT, is required. Such an extension, which is computationally very demanding, is currently under investigation.

\section{CONCLUSION}

Electronic structure calculations for bulk and surfaces with different terminations are discussed extensively in 

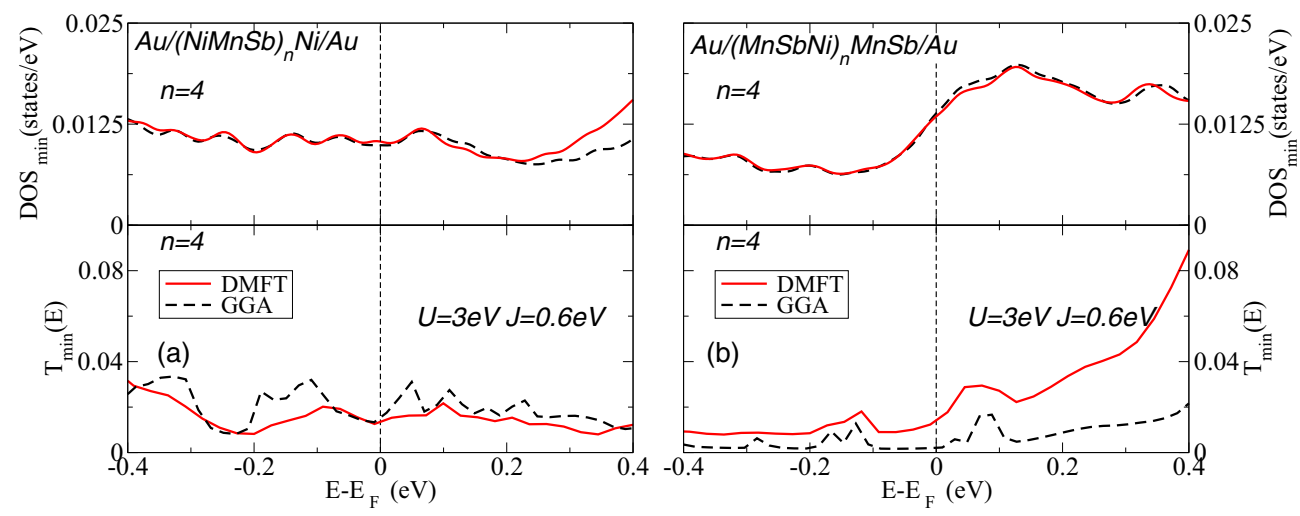

FIG. 6. Comparison of the minority spin density of states, $\mathrm{DOS}_{\min }$ (upper panel), and the minority spin transmission, $\mathrm{T}_{\min }(E)$ (lower panel). (a) Ni-terminated structure, (b) MnSb-terminated structure.

the literature $[1,2,10,64,67]$. While DMFT results for bulk $\mathrm{NiMnSb}$ have been obtained previously [2,14-16], no results including DMFT for the (001) interface and Au-capped $\mathrm{NiMnSb}$ layers have been reported before. In this work we study the effects of local electronic interactions upon the transmission across the NiMnSb layers sandwiched between gold leads. Electronic structure and transport calculations are presented using LSDA/GGA and its DMFT extension. The Au leads supply $s / p$-type orbitals in the vicinity of the Nior MnSb-terminating layers. Clearly the differences between the two interface terminations stem from the hybridization of $\mathrm{Au} s / p$ states at the interface with $\mathrm{Sb} s / p$ states. In the case of $\mathrm{MnSb}$ termination a strong hybridization is found. Similar effects are less visible for the Ni-terminated geometry. In the latter case, the $\mathrm{Sb} s / p$ states in the MnSb sublayer hybridize less with the Au $s / p$ states.

Concerning the results for transmissions, the general tendency is that electronic correlations reduce the magnitude of the transmission in the vicinity of the Fermi level. For the Niterminated structures a visible change occurs above the Fermi level in both spin channels. For the MnSb termination, a clear spin selectivity is obtained: The majority spin transmission is diminished below $E_{F}$, while minority spin transmission is enhanced above $E_{F}$. It is interesting to note that the interface states overshadow the many-body induced nonquasiparticle states. Although the spin polarization of the density of states is considerably reduced (see Fig. 3), the transmission polarization is not significantly affected by correlations (see Fig. 5). A large degree of polarization of the transmission is obtained in the case of four $\mathrm{NiMnSb}$ units, while a reduction of spin polarization down to $\approx 50 \%$ is obtained for $n=1$. We argue that this reduction is due to the direct transmission (over the scattering region) from lead to lead. Surprisingly, despite the presence of electronic correlations, there still remains a significant spin polarization in the transmission.

\section{ACKNOWLEDGMENTS}

We are grateful to Ivan Rungger for stimulating discussions. Part of the calculations were performed in the data center of NIRDIMT. Financial support offered by the Augsburg Center for Innovative Technologies, and by the Deutsche Forschungsgemeinschaft (through TRR 80) is gratefully acknowledged. C.M. thanks UEFISCDI for financial support through project PN-III-P4-ID-PCE-2016-0217. The research reported in this publication was supported by funding from King Abdullah University of Science and Technology (KAUST).
[1] R. A. de Groot, F. M. Mueller, P. G. van Engen, and K. H. J. Buschow, Phys. Rev. Lett. 50, 2024 (1983).

[2] M. I. Katsnelson, V. Y. Irkhin, L. Chioncel, A. I. Lichtenstein, and R. A. de Groot, Rev. Mod. Phys. 80, 315 (2008).

[3] B. B. W. Van Roy, J. De Boeck, and G. Borghs, Appl. Phys. Lett. 77, 4190 (2000).

[4] B. Kwon, Y. Sakuraba, H. Sukegawa, S. Li, G. Qu, T. Furubayashi, and K. Hono, J. Appl. Phys. 119, 023902 (2016).

[5] R. J. Soulen, Jr., J. M. Byers, M. S. Osofsky, B. Nadgorny, T. Ambrose, S. F. Cheng, P. R. Broussard, C. T. Tanaka, J. Nowak, J. S. Moodera, A. Barry, and J. M. D. Coey, Science 282, 85 (1998).

[6] R. Kabani, M. Terada, A. Roshko, and J. S. Moodera, J. Appl. Phys. 67, 4898 (1990).

[7] D. Ristoiu, B. B. J. P. Nozières, C. N. Borca, and P. A. Dowben, Appl. Phys. Lett. 76, 2349 (2000).
[8] C. N. Borca, T. Komesu, H.-K. Jeong, P. A. Dowben, D. Ristoiu, C. Hordequin, J. Pierre, and J. P. Nozières, Appl. Phys. Lett. 77, 88 (2000).

[9] C. N. Borca, T. Komesu, H.-K. Jeong, P. A. Dowben, D. Ristoiu, C. Hordequin, J. P. Nozières, J. Pierre, S. Stadler, and Y. U. Idzerda, Phys. Rev. B 64, 052409 (2001).

[10] M. Ležaić, P. Mavropoulos, J. Enkovaara, G. Bihlmayer, and S. Blügel, Phys. Rev. Lett. 97, 026404 (2006).

[11] H. Kolev, G. Rangelov, J. Braun, and M. Donath, Phys. Rev. B 72, 104415 (2005).

[12] M. J. Otto, H. Feil, R. A. M. V. Woerden, J. Wijngaard, P. J. V. D. Valk, C. F. V. Bruggen, and C. Haas, J. Magn. Magn. Mater. 70, 33 (1987).

[13] R. Skomski, J. Phys.: Condens. Matter 19, 315202 (2007).

[14] L. Chioncel, M. I. Katsnelson, R. A. de Groot, and A. I. Lichtenstein, Phys. Rev. B 68, 144425 (2003). 
[15] L. Chioncel, E. Arrigoni, M. I. Katsnelson, and A. I. Lichtenstein, Phys. Rev. Lett. 96, 137203 (2006).

[16] L. Chioncel, E. Arrigoni, M. I. Katsnelson, and A. I. Lichtenstein, Phys. Rev. B 79, 125123 (2009).

[17] H. Allmaier, L. Chioncel, E. Arrigoni, M. I. Katsnelson, and A. I. Lichtenstein, Phys. Rev. B 81, 054422 (2010).

[18] C. Morari, H. Allmaier, F. Beiuşeanu, T. Jurcuţ, and L. Chioncel, Phys. Rev. B 85, 085413 (2012).

[19] J. A. Caballero, Y. D. Park, J. R. Childress, J. Bass, W.-C. Chiang, A. C. Reilly, W. P. Pratt Jr., and F. Petroff, J. Vac. Sci. Technol. A 16, 1801 (1998).

[20] Z. Wen, T. Kubota, T. Yamamoto, and K. Takanashi, Sci. Rep. 5, 18387 (2015).

[21] M. I. Katsnelson and A. I. Lichtenstein, Eur. Phys. J. B 30, 9 (2002).

[22] A. I. Lichtenstein and M. I. Katsnelson, Phys. Rev. B 57, 6884 (1998).

[23] L. Chioncel, L. Vitos, I. A. Abrikosov, J. Kollar, M. I. Katsnelson, and A. I. Lichtenstein, Phys. Rev. B 67, 235106 (2003).

[24] W. Metzner and D. Vollhardt, Phys. Rev. Lett. 62, 324 (1989).

[25] A. Georges, G. Kotliar, W. Krauth, and M. J. Rozenberg, Rev. Mod. Phys. 68, 13 (1996).

[26] G. Kotliar and D. Vollhardt, Phys. Today 57, 53 (2004).

[27] L. Chioncel, C. Morari, A. Östlin, W. H. Appelt, A. Droghetti, M. M. Radonjić, I. Rungger, L. Vitos, U. Eckern, and A. V. Postnikov, Phys. Rev. B 92, 054431 (2015).

[28] A. R. Rocha, V. M. García-Suárez, S. Bailey, C. Lambert, J. Ferrer, and S. Sanvito, Phys. Rev. B 73, 085414 (2006).

[29] A. R. Rocha, V. M. García Suárez, S. Bailey, C. Lambert, J. Ferrer, and S. Sanvito, Nat. Mater. 4, 335 (2005).

[30] I. Rungger and S. Sanvito, Phys. Rev. B 78, 035407 (2008).

[31] J. M. Soler, E. Artacho, J. D. Gale, A. García, J. Junquera, and P. Ordejón, J. Phys.: Condens. Matter 14, 2745 (2002).

[32] K. Held, Adv. Phys. 56, 829 (2007).

[33] O. K. Andersen and T. Saha-Dasgupta, Phys. Rev. B 62, R16219 (2000).

[34] L. Vitos, H. L. Skriver, B. Johansson, and J. Kollár, Comput. Mater. Sci. 18, 24 (2000).

[35] L. Vitos, Phys. Rev. B 64, 014107 (2001).

[36] P. Weinberger, Electron Scattering Theory for Ordered and Disordered Matter (Clarendon Press, Oxford, 1990).

[37] J. M. Wills, M. Alouani, P. Andersson, A. Delin, O. Eriksson, and O. Grechnev, Full-Potential Electronic Structure Method (Springer, Berlin, 2010).

[38] O. Grånäs, I. D. Marco, P. Thunström, L. Nordström, O. Eriksson, T. Björkman, and J. Wills, Comput. Mater. Sci. 55, 295 (2012).

[39] R. Landauer, IBM J. Res. Dev. 1, 223 (1957).

[40] R. Landauer, IBM J. Res. Dev. 32, 306 (1988).

[41] M. Büttiker, Phys. Rev. Lett. 57, 1761 (1986).

[42] M. Büttiker, IBM J. Res. Dev. 32, 317 (1988).

[43] D. S. Fisher and P. A. Lee, Phys. Rev. B 23, 6851 (1981).

[44] J. Taylor, H. Guo, and J. Wang, Phys. Rev. B 63, 245407 (2001).

[45] K. M. Schep, P. J. Kelly, and G. E. W. Bauer, Phys. Rev. Lett. 74, 586 (1995).

[46] K. M. Schep, P. J. Kelly, and G. E. W. Bauer, Phys. Rev. B 57, 8907 (1998).

[47] W. H. Butler, X.-G. Zhang, T. C. Schulthess, and J. M. MacLaren, Phys. Rev. B 63, 054416 (2001).
[48] I. Rungger, O. Mryasov, and S. Sanvito, Phys. Rev. B 79, 094414 (2009).

[49] N. M. Caffrey, T. Archer, I. Rungger, and S. Sanvito, Phys. Rev. Lett. 109, 226803 (2012).

[50] M. Imada, A. Fujimori, and Y. Tokura, Rev. Mod. Phys. 70, 1039 (1998).

[51] G. Kotliar, S. Y. Savrasov, K. Haule, V. S. Oudovenko, O. Parcollet, and C. A. Marianetti, Rev. Mod. Phys. 78, 865 (2006).

[52] L. V. Pourovskii, M. I. Katsnelson, and A. I. Lichtenstein, Phys. Rev. B 72, 115106 (2005).

[53] N. E. Bickers and D. J. Scalapino, Ann. Phys. (NY) 193, 206 (1989).

[54] M. I. Katsnelson and A. I. Lichtenstein, J. Phys.: Condens. Matter 11, 1037 (1999).

[55] L. Chioncel, M. I. Katsnelson, G. A. de Wijs, R. A. de Groot, and A. I. Lichtenstein, Phys. Rev. B 71, 085111 (2005).

[56] D. M. Edwards and J. A. Hertz, J. Phys. F 3, 2191 (1973).

[57] H. J. Vidberg and J. W. Serene, J. Low Temp. Phys. 29, 179 (1977).

[58] A. Östlin, L. Chioncel, and L. Vitos, Phys. Rev. B 86, 235107 (2012).

[59] A. I. Lichtenstein, M. I. Katsnelson, and G. Kotliar, Phys. Rev. Lett. 87, 067205 (2001).

[60] A. G. Petukhov, I. I. Mazin, L. Chioncel, and A. I. Lichtenstein, Phys. Rev. B 67, 153106 (2003).

[61] A. Oguri, J. Phys. Soc. Jpn. 70, 2666 (2001).

[62] Y. Meir and N. S. Wingreen, Phys. Rev. Lett. 68, 2512 (1992).

[63] J. P. Perdew and Y. Wang, Phys. Rev. B 45, 13244 (1992).

[64] I. Galanakis, P. H. Dederichs, and N. Papanikolaou, Phys. Rev. B 66, 134428 (2002).

[65] P. Mavropoulos, K. Sato, R. Zeller, P. H. Dederichs, V. Popescu, and H. Ebert, Phys. Rev. B 69, 054424 (2004).

[66] J. P. Perdew, K. Burke, and M. Ernzerhof, Phys. Rev. Lett. 77, 3865 (1996).

[67] S. Jenkins and D. King, Surf. Sci. 494, L793 (2001).

[68] S. Plogmann, T. Schlathölter, J. Braun, M. Neumann, Yu. M. Yarmoshenko, M. V. Yablonskikh, E. I. Shreder, E. Z. Kurmaev, A. Wrona, and A. Ślebarski, Phys. Rev. B 60, 6428 (1999).

[69] M. Ležaić, I. Galanakis, G. Bihlmayer, and S. Blügel, J. Phys.: Condens. Matter 17, 3121 (2005).

[70] I. Galanakis, J. Phys.: Condens. Matter 14, 6329 (2002).

[71] M. M. Kirillova, A. A. Makhnev, E. I. Shreder, V. P. Dyakina, and N. B. Gorina, Phys. Status Solidi B 187, 231 (1995).

[72] A. Yamasaki, L. Chioncel, A. I. Lichtenstein, and O. K. Andersen, Phys. Rev. B 74, 024419 (2006).

[73] M. I. Katsnelson and D. M. Edwards, J. Phys.: Condens. Matter 4, 3289 (1992).

[74] V. Y. Irkhin and M. I. Katsnelson, Eur. Phys. J. B 43, 479 (2005).

[75] V. Y. Irkhin and M. I. Katsnelson, Phys. Rev. B 73, 104429 (2006).

[76] L. Chioncel, Y. Sakuraba, E. Arrigoni, M. I. Katsnelson, M. Oogane, Y. Ando, T. Miyazaki, E. Burzo, and A. I. Lichtenstein, Phys. Rev. Lett. 100, 086402 (2008).

[77] L. Chioncel, P. Mavropoulos, M. Ležaić, S. Blügel, E. Arrigoni, M. I. Katsnelson, and A. I. Lichtenstein, Phys. Rev. Lett. 96, 197203 (2006).

[78] F. Beiuşeanu, C. Horea, E.-V. Macocian, T. Jurcuţ, L. Vitos, and L. Chioncel, Phys. Rev. B 83, 125107 (2011).

[79] L. Chioncel, I. Leonov, H. Allmaier, F. Beiuşeanu, E. Arrigoni, T. Jurcuţ, and W. Pötz, Phys. Rev. B 83, 035307 (2011). 